\title{
TACs, ARCs and Project Process at EWB-USA
}

\author{
David Sacco, P.E. \\ Engineers Without Borders USA Mentor \\ Yale University Student Chapter \\ TPA Design Group \\ 85 Willow Street \\ New Haven, CT 06511 \\ dsacco@tpadesigngroup.com \\ 203-640-9944
}

\author{
Joshua Knight, P.E., Ph. D. \\ Engineers Without Borders USA, Project Engineer \\ joshua.knight@ewb-usa.org \\ 303-772-2723
}

\begin{abstract}
Engineers Without Borders USA has been operating and evolving since 2002. As an organization with many student and professional chapters working around the world, EWBUSA has put in place structures to review proposed community programs as well as specific engineering projects. The Application Review Committee, or ARC, considers new program proposals put forward by communities, while Technical Advisory Committees, or TACs, evaluate implementation plans for infrastructure projects. ARC and TAC members are volunteers with relevant technical knowledge and experience, many of whom are also EWBUSA chapter members. EWB-USA technical and chapter relations staff review progress at key points in the project cycle; however, this paper reviews the mainly volunteer efforts at two important moments in the project process.
\end{abstract}

Index - EWB, Service Learning Engineering, Community-centered, Quality Control, Peer Review

\section{The Application Review Committee (ARC)}

The Application Review Committee is made up of volunteer members and EWB-USA staff with relevant experience in engineering, international development or both. Each new application from a community seeking assistance is assigned to an ARC team consisting of three persons: a volunteer ARC lead with extensive experience reviewing new applications, a volunteer member of EWB-USA, and an EWB-USA staff member. The team receives the program application, provides guidance on specific aspects of the application, and decides whether to approve or decline each application.

Over time, the application process itself has shifted from project-oriented to program-centered. The difference is crucial; rather than soliciting applications for specific engineered projects such as road building, water supply or solar power, EWB-USA is seeking to establish relationships with developing communities, and to collaborate with those communities to determine how the engineering skills of a chapter might be applied to address local needs. An EWB-USA chapter is expected to make a minimum five-year commitment to a community to enhance the likelihood that any specific intervention will have met its objectives and be durable and useful over time. Each chapter will make several visits to its partner community over the course of its engagement; first assessing problems, then implementing solutions, and finally monitoring project outcomes.

Because the focus has shifted to community-identified needs rather than specific infrastructure projects, the application and review process is now directed towards establishing the capacity of an applicant community, in conjunction with its NGO or governmental partners, to communicate with a chapter and provide logistical support for visits, as well as the local and regional availability of material, labor support, 
technical oversight and travel logistics. Individual project proposals are typically included in the application, but acceptance of a program application does not imply approval of a specific project. That decision must be made by the community and the adopting chapter together based upon the findings of assessment visits and consultation with the community.

Establishing an EWB-USA program implies more than agreeing to build a school, well or clinic. It commits the chapter and host community to a collaboration that includes technical input for specific projects, educational efforts to enhance the efficacy and sustainability of projects and a shared commitment to financing project implementation, maintenance and operation. The "program" also encompasses the process of assessing local needs and resources, and consulting with the host community as broadly as possible; as well as post-implementation monitoring and evaluation to capture beneficial outcomes, ensure client satisfaction and verify proper operation and maintenance.

A program centered on a water supply project, for example, might include educational outreach to school or community groups on proper water handling and storage, the water-related disease cycle and good hygiene and sanitation practices. A chapter could start a parallel project to implement or improve latrines to further enhance health outcomes. Monitoring and evaluation of efforts might capture water quality and quantity, but also investigate family health or school attendance as secondary indicators of positive outcomes. Training for system operators and development of maintenance manuals would be key program activities, as would establishing local management for the water system.

Given this range of tasks and the limited ability of chapters to travel, EWB-USA expects other partners in the program to share the effort. These might be local governments or their technical departments, or nongovernmental organizations working in the area. Either domestic or international NGOs might be engaged; chapter partners have included municipal councils, religious organizations, alumni associations, Rotary chapters or charitable groups. Ideally, both governmental and non-governmental partners will be identified; once chapters begin making assessment visits, the roles of the partners can be clarified and additional connections might be made to ensure continuity of support and communications in both directions.

As always, finances must be considered. Chapters often provide significant monetary support for project implementation, but they are not permitted to fund operations or maintenance; and communities are expected to contribute financially as well as through labor and material support for projects. In addition, an applicant community must commit to long-term financial responsibility for operations and maintenance of completed work. These expectations frustrate some EWB-USA chapters as well as their partner communities, but the history of development efforts is littered with well-intended projects that failed for lack of parts, fuel or a continuing community commitment to properly operating and maintaining the completed project.

The job of an ARC team, then, is to consider whether all of the pieces are present so that a positive outcome is likely. Is there a defined community seeking assistance, is it committed to a partnership, will they maintain contact with and support visits by an EWB-USA chapter, and will they have the capacity to operate and maintain the proposed projects? Have NGO or governmental resources been identified to support the effort and facilitate the relationship between the community and a chapter?

To emphasize EWB-USA's focus on community-driven programs, the application process was modified in 2011 to require the community to be the applicant rather than a party to an application by a chapter. Although communities have always been able to apply for programs, many applications had been submitted by chapters or by NGOs on behalf of communities. With the new process, chapters or NGOs may still assist in the process, but they can no longer lead it. Application information and review criteria fall into a few broad categories: 
- What is the identified problem, and why is it important to the community?

- What projects are being considered, and are they feasible and affordable for a chapter to undertake?

- Who are the partners and what are their capacities?

- Does the community have the ability to communicate reliably with a chapter, and is it safe and practical for a chapter to visit?

- Is the community committed to execution of the program and to its long-term sustainability?

ARC reviews often involve follow-up questions from the panel to the community to resolve concerns or clarify situations. Not all reviews are resolved successfully, however; some common reasons for declining an application include:

- The beneficiary community is too large or ill-defined, or it excludes some local inhabitants. Occasionally, program applications are for groups of several thousand persons over large geographic areas. In these cases, it may not be clear with whom a chapter is partnering or if community members would feel connected to the program at all.

- Lack of appropriate partners such as NGOs, community-based committees, or municipal governments. Local partners are critical to maintain communications, support visits and implementation, and provide program support through capacity-building and training.

- Overemphasis on a specific project or technology can imply to chapters that the project has already been identified and designed, so that there may not be a discernible technical role for a chapter to play in a program. A project may not be adopted if chapters do not see how their technical abilities might be utilized, or a chapter might lose its enthusiasm if it feels that it is only a financial resource.

- Insufficient commitment from the community to financial support for implementation and upkeep.

In summary, the ARC serves as a gatekeeper to help ensure that communities are motivated and prepared to partner with EWB-USA chapters, and that programs are well-defined, potentially appealing to chapters seeking new opportunities, and consistent with the EWB-USA mission to support communitydriven development programs through implementation of sustainable engineering projects.

\section{The Technical Advisory Committee (TAC)}

Since EWB-USA chapters work in a wide range of settings and technical fields, the TACs were introduced to provide consistent quality control at a critical juncture in the project cycle: immediately prior to starting implementation. The principal tasks of a TAC are to decide whether or not a proposed project would be beneficial to the partner community and whether or not that project could be safely and practically built by the chapter and community in partnership. In addition, TAC members provide suggestions based on their professional experience to enhance project quality and feasibility.

The TAC process also helps to introduce student chapters to the concept of peer review that is integral to professional engineering practice. For professional chapters, TAC members can provide insights on the application of domestic experience to different cultural and physical settings. 
TAC members represent a broad range of experience and specialization from electrical engineering to bridge design, from humanitarian relief to hydraulic modeling. While each panelist brings his or her special knowledge to bear on certain reviews, it is equally important that they apply common sense and personal experience as well as professional expertise. Sometimes projects are found to be too ambitious for the available time, unnecessarily complex, or too ill-defined. TACs may also address community participation, appropriateness of selected techniques or approaches, or sustainability as well as specific engineering concerns. In the TAC context, "sustainability" refers to the likelihood that the constructed work will serve its intended function over an extended period of time given regular maintenance that can be accomplished by the community using locally available tools and materials along with training provided by the chapter.

TACs focus on the specific implementation activities that are proposed by chapters following site assessment and community consultation. TACs are regionally-based, largely to accommodate chapters and volunteer reviewers in four different time zones spanning the United States. TAC members are volunteers, most hold a Professional Engineering or similar credential, and many have international development experience through EWB-USA or other organizations.

All implementation plans are submitted for review by EWB-USA project engineers at two stages. First, an alternatives analysis is prepared to document how the findings of an assessment trip led to a chapter's chosen strategy for a project. For example, an unsafe water supply might be addressed by filtration, treatment, or identification of an alternative source. Each option would have costs and benefits and at this stage the chapter would review its decision-making process with a staff project engineer.

Next, a chapter would develop its chosen alternative and prepare a preliminary design report for staff review. The focuses at this step are to ensure that a chapter is providing adequate detail in its design and calculations, and is taking community input into account. Finally, a completed design report with supporting calculations and drawings is submitted for TAC review.

TACs meet once or twice monthly. TAC members receive the design reports a week in advance of each meeting and consider up to three proposals at each meeting. Each session is a "webinar" and conference call; chapter presenters, EWB-USA project engineers and TAC panelists log into a website to view chapter presentations and deliberate over a conference phone line. Chapters have twenty minutes to present followed by ten minutes of question-and-answer from the TAC panel; after the chapters log out, the TAC deliberates and approves or declines each proposal. In some cases approvals are conditioned upon submission of additional information by a chapter. TAC approval is required for travel authorization.

TAC members may consider the following in their reviews and deliberations:

- Has the design been adequately documented in plans, details and specifications?

- Is the design fully supported by field data and engineering calculations?

- Is the construction process feasible for a chapter and its partners?

- Are the technologies and materials locally available and appropriate for the need and setting?

- Will the finished project be durable, operable and readily maintained using local finances, materials and technicians?

- In addition, a Health and Safety Plan developed for the specific project type and location must be reviewed and approved by a TAC member with training and experience in such reviews.

Some projects are approved by the TAC without conditions, but many are approved with conditions and some are declined. Typically, declined implementation trips are repurposed as assessment missions to gain additional information to improve designs and address TAC concerns. 
Some common problems identified by TACs include:

- Lack of clarity as to trip objectives. Sometimes chapters have not obtained sufficient data during assessments, or fully addressed community concerns. They may expect to resolve these problems at the beginning of an implementation visit, but TACs generally discourage this unless the issues (and their impacts) are minor.

- Insufficient information in drawings. Ideally, a design report should be self-sufficient, so that a competent and experienced contractor could execute the work with limited oversight from the chapter.

- Overly ambitious scheduling. Chapters often underestimate the practical and logistical challenges of working in developing-world rural communities, and expect to accomplish an unrealistic amount of work in a few weeks. This problem can often be addressed by phasing the implementation over several trips and identifying local engineering technicians at NGOs or government departments who might provide ongoing oversight between chapter visits.

- Inappropriate technology. Chapters sometimes look to new or innovative methods or materials that may not be available locally or may not be maintainable in the long run. Chapters are required to source all materials in-country and as locally as practical. Applying local material, skills and techniques enhances sustainability and increases the economic impact of projects.

- Inadequate consideration of critical details. If an area is earthquake prone, has seismicity been considered in the design of structures? When working in watercourses, have channel stability and flooding been considered? Has erosion been considered at culverts, piers or drainage outfalls?

The TAC process generally runs smoothly, but as with any decision-making body there may be differences of opinion within the panel. TACs try to reach consensus on each decision, but that sometimes fails and the majority opinion prevails. Given the very short time allotted for questions and discussion, some TAC members do not have an opportunity to raise all of their concerns and may not feel ready to grant an approval.

A bigger issue is that chapters are sometimes unhappy with the TAC decision and in those cases are usually quite surprised to have been declined. This is most often true with university chapters, since students graduate, collective experience often leaves with them, and new members may not understand what the TAC expects from design reports and presentations. Similarly, faculty and professional mentors may not serve for more than a few years, and new mentors take time to grasp the process.

That being said, chapters put in a tremendous (if varied) amount of effort to raise funds and prepare designs, and mentors take a lot of time off work and away from family to support projects. TAC members are expected to show appropriate respect for each chapter's commitment and effort and to keep comments constructive. It is our responsibility to point out how designs and presentations could be improved, but each decision must be made by assessing the crucial elements of a proposal and determining whether or not the chapter has gotten it right. Regardless of the quality of presentations, part of the TAC member's job is to help each chapter understand what a well-conceived project looks like, and to help chapter members find ways to move forward, gain experience and build connections with their communities.

Whether or not the TAC panel would have made the same design decisions as a chapter has made, it is our duty to respect the effort that has been expended and to focus on making the proposed implementation 
as successful as possible through our comments. We seek to be part of each chapter's learning process by making focused, constructive comments, and showing how peer review can lead to good engineering work.

In the end, the point of the TAC is to protect the interests of communities seeking assistance and chapters providing guidance by ensuring that a careful and deliberate design process has been followed in consultation with the beneficiary community, and that proposed work is likely to be safe, feasible, functional and beneficial.

\section{Conclusion}

As the EWB-USA process has evolved, two structures have been created to harness the technical expertise of volunteer member engineers and make it available to EWB-USA chapters: the Application Review Committee and the Technical Advisory Committees. These groups, working closely with EWB-USA staff, have become important tools in improving project quality and effectiveness, as well as presenting rewarding opportunities to engineers with an interest in human development.

This paper reflects the efforts of EWB-USA staff and volunteers over the past decade to develop, review and revise effective processes for community development. We are grateful for the opportunity to synthesize and present the work of the many people who have contributed to this task and wish to acknowledge their contributions. 